\title{
Disease status, reasons for discontinuation and adverse events in 1038 Italian children with juvenile idiopathic arthritis treated with etanercept
}

Sara Verazza', Sergio Davi², Alessandro Consolaro ${ }^{1,2}$, Francesca Bovis², Antonella Insalaco ${ }^{3}$, Silvia Magni-Manzoni ${ }^{3}$, Rebecca Nicolai ${ }^{3}$, Denise Pires Marafon ${ }^{3}$, Fabrizio De Benedetti ${ }^{3}$, Valeria Gerloni ${ }^{4}$, Irene Pontikaki ${ }^{4}$,

Francesca Rovellii, Rolando Cimaz ${ }^{5}$, Achille Marino ${ }^{5}$, Francesco Zulian ${ }^{6}$, Giorgia Martini ${ }^{6}$, Serena Pastore ${ }^{7}$,

Chiara Sandrin ${ }^{7}$, Fabrizia Corona $^{8}$, Marta Torcoletti ${ }^{8}$, Giovanni Conti $^{9}$, Claudia Fede ${ }^{9}$, Patrizia Barone ${ }^{10}$, Marco Cattalini ${ }^{11}$, Elisabetta Cortis ${ }^{12}$, Luciana Breda ${ }^{13}$, Alma Nunzia Olivieri ${ }^{14}$, Adele Civino ${ }^{15}$, Rosanna Podda ${ }^{16}$, Donato Rigante ${ }^{17}$, Francesco La Torre ${ }^{18}$, Gianfranco D'Angelo ${ }^{19}$, Mauro Jorini ${ }^{20}$, Romina Gallizzi ${ }^{21}$, Maria Cristina Maggio ${ }^{22}$, Rita Consolini ${ }^{23}$, Alessandro De Fantii ${ }^{24}$, Valentina Muratore ${ }^{25}$, Maria Giannina Alpigiani ${ }^{2}$, Nicolino Ruperto ${ }^{2}$, Alberto Martini ${ }^{1,2}$, Angelo Ravelli $^{1,2,26^{*}}$ and on behalf of the Italian Pediatric Rheumatology Study Group

\section{Abstract}

Background: Data from routine clinical practice are needed to further define the efficacy and safety of biologic medications in children with juvenile idiopathic arthritis (JIA). The aim of this analysis was to investigate the disease status, reasons for discontinuation and adverse events in Italian JIA patients treated with etanercept (ETN).

Methods: In 2013, all centers of the Italian Pediatric Rheumatology Study Group were asked to make a census of patients given ETN after January 2000. Patients were classified in three groups: group $1=$ patients still taking ETN; group 2 = patients discontinued from ETN for any reasons; group 3 = patients lost to follow-up while receiving ETN. All three groups received a retrospective assessment; patients in group 1 also underwent a cross-sectional assessment.

Results: 1038 patients were enrolled by 23 centers: 422 (40.7\%) were in group 1, 462 (44.5\%) in group 2, and 154 (14.8\%) in group 3. Median duration of ETN therapy was 2.5 years. At cross-sectional assessment, $41.8 \%$ to $48.6 \%$ of patients in group 1 met formal criteria for inactive disease, whereas 52.4\% of patients in group 2 and $55.8 \%$ of patients in group 3 were judged in clinical remission by their caring physician at last visit. A relatively greater proportion of patients with systemic arthritis were discontinued or lost to follow-up. Parent evaluations at cross-sectional visit in group 1 showed that $52.4 \%$ of patients had normal physical function, very few had impairment in quality of life, $51.2 \%$ had no pain, $76 \%$ had no morning stiffness, and $82.7 \%$ of parents were satisfied with their child's illness outcome. Clinically significant adverse events were reported for $27.8 \%$ of patients and ETN was discontinued for side effects in $9.5 \%$. The most common adverse events were new onset or recurrent uveitis (10.2\%), infections (6.6\%), injection site reactions (4.4\%), and neuropsychiatric (3.1\%), gastrointestinal (2.4\%), and hematological disorders (2.1\%). Ten patients developed an inflammatory bowel disease and 2 had a malignancy. One patient died of a fulminant streptococcal sepsis.

(Continued on next page)

\footnotetext{
* Correspondence: angeloravelli@gaslini.org

${ }^{1}$ Università degli Studi di Genova, Genova, Italy

${ }^{2}$ Istituto Giannina Gaslini, Genova, Italy

Full list of author information is available at the end of the article
} 
(Continued from previous page)

Conclusions: Around half of the patients achieved complete disease quiescence under treatment with ETN. The medication was overall well tolerated, as only one quarter of patients experienced clinically significant adverse events and less than $10 \%$ had treatment discontinued for toxicity.

Keywords: Juvenile idiopathic arthritis, Etanercept, Pediatric rheumatology, TNF inhibitors, Biologic therapies

\section{Background}

Etanercept (ETN), a tumor necrosis factor (TNF) antagonist, has been the first biologic agent registered for use in children with juvenile idiopathic arthritis (JIA). Its efficacy and safety have been established in a randomized placebo-controlled withdrawal trial in patients with a polyarticular disease course who were refractory or intolerant to methotrexate (MTX) [1]. Long-term extension studies of the original trial cohort and national registries have subsequently confirmed the sustained clinical benefit and acceptable tolerability of the drug [2-5]. The evidence for the effectiveness of ETN in JIA has been expanded by the observation that its administration is associated with improvement of functional ability and health-related quality of life [6-8], recovery of growth velocity and bone status $[9,10]$, and inhibition of progression of radiographic joint damage [11]. Recent studies have shown that around half of children with JIA who are treated with ETN in clinical practice are able to attain complete disease quiescence [12-14]. However, there is a need of more data from large series of patients treated in real-life clinical settings to fully characterize the efficacy and safety profile of this medication.

In June 2013, the Italian Pediatric Rheumatology Study Group undertook a national multicenter survey aimed to investigate the disease status, reasons for discontinuation and adverse events in Italian patients with JIA who were receiving or had received ETN. The findings of this study, named Etanercept in Italian Children with Arthritis (EtICA) study, are described in the present article.

\section{Methods}

\section{Study design and patient enrolment}

All centers that are part of the Italian Pediatric Rheumatology Study Group were invited to participate in this multicenter, observational study. To minimize a selection bias, investigators at each center were first asked to make a census of all patients with JIA by the International League of Associations for Rheumatology (ILAR) criteria [15] who were given ETN at the center after January 2000 and had received the medication for at least 6 months. Based on the status of ETN therapy at the time of the census, patients were classified in three groups: group $1=$ patients still taking ETN; group 2 patients discontinued from ETN for any reasons; group
3 patients lost to follow-up while receiving ETN. Next, each investigator was asked to make a retrospective assessment for all patients in groups 1, 2 and 3, and a cross-sectional assessment at first visit after the census for all patients in group 1. Study data were collected in ad hoc case reports forms. Once completed, all forms were sent to the coordinating center (the Gaslini Institute, Genoa, Italy). Ethics committee approval of the study protocol was obtained in all participating centers.

\section{Retrospective assessment}

The following information was collected through the review of patients charts: sex, age at disease onset, ILAR category, disease duration at first visit at study center and at start of ETN, medications administered before ETN start and during ETN therapy, ETN-related side effects, duration of ETN therapy at treatment discontinuation (for patients in group 2) or at last follow-up visit (for patients in group 3), reasons for ETN discontinuation (for patients in group 2), and disease activity state (remission or active disease) at last follow-up visit (for patients in group 3).

\section{Cross-sectional assessment}

The following clinical evaluations were performed at cross-sectional visit in patients still taking ETN (group 1). The caring physician made a standardized joint examination and recorded the count of joints with swelling, pain/tenderness, restricted motion, and active arthritis [16]; in addition, he or she also rated the overall disease activity on a 21-numbered circle visual analog scale (VAS; where $0=$ no activity and $10=$ maximum activity) [17]. The same physician also assessed the amount of articular and extra-articular damage through the Juvenile Arthritis Damage Index (JADI) [18]. Briefly, the JADIArticular (JADI-A) assesses 36 joints or joint groups for the presence of damage, and the damage observed in each joint is scored on a 3-point scale $(0=$ no damage, $1=$ partial damage, and $2=$ severe damage, ankylosis, or prosthesis). The maximum total score is 72 . The JADIExtraarticular (JADI-E) includes 13 items in 5 different organs/systems. Each item is scored as 0 if damage is absent or as 1 if damage is present. Due to the relevant impact of ocular damage on the child's health, in each eye a score of 2 is given in case the patient has had ocular 
surgery, and a score of 3 is given in case the patient has developed legal blindness. The maximum total score is 17 .

Prior to the physician visit, a parent (or guardian) completed the parent version of the Juvenile Arthritis Multidimensional Questionnaire (JAMAR) [19]. This questionnaire incorporates all main parent-centered outcome measures, including physical function, healthrelated quality of life (HRQL), overall well-being, pain, level of disease activity, self- or proxy-reported joint count, morning stiffness, assessment of disease status and course, side effects of medications, therapeutic compliance, difficulties at school, and satisfaction with illness outcome. We previously found that the mean (SD) HRQL score in 801 healthy children was 2.6 (2.4) and that the mean (SD) score of the physical health and psychosocial health subscales was 0.8 (1.2) and 1.8 (1.7), respectively (Bertamino $\mathrm{M}$ et al. Unpublished observation).

Laboratory investigations included the erythrocyte sedimentation rate (ESR) and the $C$-reactive protein (CRP), but were performed only if deemed necessary by the caring physician for clinical purposes.

Informed consent to participate in the study was provided by both the parent/guardian and the patient (when applicable) for all patients who underwent the cross-sectional assessment.

\section{Definitions of disease activity states}

In patients still taking ETN (group 1), the state of disease activity at cross-sectional visit was assessed through the following criteria: 1) criteria for inactive disease (ID) in JIA [20]; 2) preliminary definition of minimal disease activity in JIA [21]; 3) criteria for inactive disease and low disease activity (LDA), moderate disease activity (MDA), and high disease activity (HDA) in JIA based on the Juvenile Arthritis Disease Activity Score (JADAS) and the clinical JADAS (cJADAS) [22-24]. To harmonize the terminology of disease activity states with that used in the adult rheumatology community, in this study, the term minimal disease activity has been replaced with LDA, which has an identical meaning. For patients in groups 2 and 3, the disease activity state at treatment discontinuation or last follow-up visit, respectively, was defined by the caring physician based on the data recorded in the clinical chart.

\section{Recording of adverse events}

ETN-related adverse events were recorded for all patients in groups 1,2 and 3. Study investigators were instructed to report all adverse events that they considered clinically significant, without pre-specified definitions of characteristics and severity. Adverse events that led to treatment discontinuation were registered for patients who had ETN discontinued for side effects.

\section{Statistics}

Descriptive statistics are reported as median and interquartile range (IQR) for continuous variables and as absolute frequency and percentage for categorical variables. Comparisons of disease characteristics between patient groups were performed by means of Mann-Whitney $U$ test in case of quantitative data and by means of the chisquare test, or the Fisher's Exact test, as appropriate, in case of categorical data. All statistical tests were twosided; a $P$ value of less than 0.05 was considered as statistically significant. The statistical package used were SAS 9.3 (Institute Inc., Cary, NC, USA) and Statistica (version 8.0, StatSoft Corp., Tulsa, OK).

\section{Results}

All 26 invited pediatric rheumatology centers agreed to participate in the study and included in the census a total of 1231 patients. However, 3 centers, which had listed 109 patients, did not provide any evaluations and were then excluded. Of the remaining 1122 patients, 1068 (95.2\%) underwent the study assessments. Thirty of them were enrolled by more than one center and were, thus, counted only once (by retaining the most recent assessment), leading to a final number of assessed patients of 1038. Of them, $422(40.7 \%)$ were still receiving ETN (group 1), 462 (44.5\%) had been discontinued from ETN (group 2), and 154 (14.8\%) had been lost to follow-up while receiving ETN (group 3). For the purpose of the analyses, patients in group 1 were placed in a "cross-sectional group" and patients in groups 2 and 3, who received only retrospective assessments, were combined in a single "retrospective group".

The characteristics of the study patients at the start of ETN therapy are presented in Table 1. Overall, the study cohort was characterized by marked female prevalence, young age at onset, and high frequency of ANA positivity. The median disease duration (3.5 years) was quite long, but the average duration of follow-up at the study center was 2.1 years. Around two third of patients had extended oligoarthritis or rheumatoid factor-negative polyarthritis. Patients with persistent oligoarthritis outnumbered those with psoriatic arthritis, enthesitis-related arthritis, or undifferentiated arthritis. There were more patients with systemic arthritis in the retrospective group than in the cross-sectional group. Patients in the crosssectional group had a younger age at disease onset and were more frequently ANA positive than those in the retrospective group. Before the start of ETN, approximately $90 \%$ of the patients had received MTX, $50 \%$ systemic corticosteroids, and 55\% intra-articular corticosteroids. 53 patients were given biologic medications other than ETN before treatment start, which included adalimumab $(n=20)$, infliximab $(n=19)$, anakinra $(n=15)$, abatacept $(n=3)$, and tocilizumab 
Table 1 Baseline characteristics of children with juvenile idiopathic arthritis treated with etanercept ${ }^{\mathrm{a}}$

\begin{tabular}{|c|c|c|c|c|}
\hline & $\begin{array}{l}\text { All patients } \\
(n=1038)\end{array}$ & $\begin{array}{l}\text { Cross-sectional group }^{\mathrm{b}} \\
(n=422)\end{array}$ & $\begin{array}{l}\text { Retrospective group }{ }^{c} \\
(n=616)\end{array}$ & $P$ \\
\hline Female & $781(75.2)$ & $335(79.4)$ & $446(72.4)$ & 0.01 \\
\hline \multicolumn{5}{|l|}{ ILAR category } \\
\hline Systemic arthritis & $106(10.2)$ & $21(5)$ & $85(13.8)$ & $<0.0001$ \\
\hline Persistent oligoarthritis & $139(13.4)$ & $60(14.2)$ & 79 (12.8) & 0.52 \\
\hline Extended oligoarthritis & $325(31.3)$ & $147(34.8)$ & $178(28.9)$ & 0.04 \\
\hline RF-negative polyarthritis & $329(31.7)$ & $138(32.7)$ & $191(31)$ & 0.56 \\
\hline RF-positive polyarthritis & $50(4.8)$ & $16(3.8)$ & $34(5.5)$ & 0.20 \\
\hline Enthesitis-related arthritis & $48(4.6)$ & $21(5)$ & $27(4.4)$ & 0.65 \\
\hline Psoriatic arthritis & $34(3.3)$ & $16(3.8)$ & $18(2.9)$ & 0.44 \\
\hline Undifferentiated arthritis & $7(0.7)$ & $3(0.7)$ & $4(0.6)$ & 1.0 \\
\hline Patients with positive ANA & $586 / 1028(57)$ & $278(66.5)$ & $308(50.5)$ & $<0.0001$ \\
\hline Median (IQR) age at disease onset, years & $4.1(2-8.3)$ & $3.5(1.8-7.3)$ & $4.7(2.1-9.1)$ & 0.001 \\
\hline Median (IQR) age, years & $10.1(6.2-13.9)$ & $9.6(5.8-13.6)$ & $10.4(6.9-14.2)$ & 0.04 \\
\hline Median (IQR) disease duration, years & $3.5(1.3-7.5)$ & $3.6(1.4-7.8)$ & $3.3(1.3-7.3)$ & 0.16 \\
\hline Patients aged $<4$ years & $122(11.8)$ & $54(12.8)$ & $68(11)$ & 0.39 \\
\hline Median (IQR) follow-up duration ${ }^{d}$, years & $2.1(0.6-5.5)$ & $2.4(0.7-6.3)$ & $2.1(0.5-5.1)$ & 0.04 \\
\hline \multicolumn{5}{|l|}{ Treatments before ETN start } \\
\hline NSAIDs & $603(58.1)$ & $269(63.7)$ & $334(54.2)$ & 0.002 \\
\hline Systemic corticosteroids & $527(50.8)$ & $198(46.9)$ & $329(53.4)$ & 0.04 \\
\hline Intra-articular corticosteroids & $579(55.8)$ & $263(62.3)$ & $316(51.3)$ & 0.0004 \\
\hline Methotrexate & $930(89.6)$ & $387(91.7)$ & $543(88.1)$ & 0.07 \\
\hline Other synthetic DMARDs & $187(18)$ & $48(11.4)$ & $139(22.6)$ & $<0.0001$ \\
\hline Other biologic agents & $53(5.1)$ & $18(4.3)$ & $35(5.7)$ & 0.31 \\
\hline \multicolumn{5}{|c|}{ Concomitant therapies during ETN administration } \\
\hline Systemic corticosteroids & $267(25.7)$ & $93(22)$ & $174(28.2)$ & 0.02 \\
\hline Intra-articular corticosteroids & $257(24.8)$ & $98(23.2)$ & $159(25.8)$ & 0.34 \\
\hline Methotrexate & $749(72.2)$ & $311(73.7)$ & $438(71.1)$ & 0.36 \\
\hline Other synthetic DMARDs & $73(7)$ & $17(4)$ & $56(9.1)$ & 0.002 \\
\hline
\end{tabular}

ILAR International League of Associations for Rheumatology, ANA antinuclear antibodies, ETN etanercept, IQR interquartile range, NSAIDs nonsteroidal anti-inflammatory drugs, DMARDs disease-modifying antirheumatic drugs

${ }^{\text {aD }}$ ata are number (percentage) unless otherwise indicated

${ }^{b}$ Includes patients still receiving ETN

Includes patients discontinued from ETN or lost to follow-up

${ }^{\mathrm{d} D u r a t i o n}$ of follow-up at study center

$(n=1)$. During ETN therapy, more than $70 \%$ of the patients were given concomitant MTX therapy and around $25 \%$ corticosteroids, either systemic or intraarticular. The median (IQR) duration of ETN therapy in the cross-sectional and retrospective group was 2.5 (1-4.2) years and $2.6(1.3-4.4)$ years, respectively. $79.5 \%$ of patients were treated for at least 1 year, $58.5 \%$ for at least 2 years, and $19.1 \%$ for at least 5 years.

Table 2 shows the disease activity states and concomitant therapies at cross-sectional visit in patients still receiving ETN (cross-sectional group). The percentage of patients who met the Wallace criteria for ID [20] and the Magni-Manzoni criteria for LDA (or minimal disease activity) [21] was 41.8 and $63.6 \%$, respectively. Notably, the exclusion of the criterion that requires the normality of acute phase reactants from Wallace criteria led to increase the proportion of patients with ID to $51 \%$. The percentage of patients who met the JADAS10 criteria for ID, LDA, MDA and HDA [22, 23] was 46.4, 64.0, 24.4, and $11.6 \%$, respectively. The figures obtained for the cJADAS10 [24] were similar. $68.5 \%$ of patients had no active joints and $56.6 \%$ had a physician global assessment indicating no disease activity. More than $80 \%$ of patients had normal ESR or CRP. 77.3 and $86.9 \%$ of patients had no evidence of articular or extra-articular damage, respectively. As compared to treatment baseline, 
Table 2 Therapeutic data and disease activity states at cross-sectional visit in patients still receiving etanercept $(n=422)^{a}$

\begin{tabular}{|c|c|c|}
\hline & No assessed & No (\%) positive \\
\hline \multicolumn{3}{|l|}{ Concomitant therapies } \\
\hline Systemic corticosteroids & 422 & $20(4.7)$ \\
\hline Intra-articular corticosteroids & 422 & $11(2.6)$ \\
\hline Methotrexate & 422 & $200(47.4)$ \\
\hline Salazopyrin & 422 & $3(0.7)$ \\
\hline Azathioprine & 422 & $1(0.2)$ \\
\hline \multicolumn{3}{|l|}{ Disease activity states - Formal definitions } \\
\hline Wallace criteria for inactive disease & 392 & $164(41.8)$ \\
\hline $\begin{array}{l}\text { Wallace criteria for inactive disease without } \\
\text { acute phase reactants }\end{array}$ & 420 & $214(51)$ \\
\hline Magni-Manzoni criteria for low disease activity & 420 & $267(63.6)$ \\
\hline \multicolumn{3}{|l|}{ Disease activity states - JADAS10 } \\
\hline Inactive disease & 422 & $196(46.4)$ \\
\hline Low disease activity ${ }^{b}$ & 422 & $270(64)$ \\
\hline Moderate disease activity & 422 & $103(24.4)$ \\
\hline High disease activity & 422 & 49 (11.6) \\
\hline \multicolumn{3}{|l|}{ Disease activity states - cJADAS10 } \\
\hline Inactive disease & 422 & $205(48.6)$ \\
\hline Low disease activity ${ }^{b}$ & 422 & $246(58.3)$ \\
\hline Moderate disease activity & 422 & $117(27.7)$ \\
\hline High disease activity & 422 & $59(14)$ \\
\hline Patients with no active joints & 422 & $289(68.5)$ \\
\hline Patients with no swollen joints & 422 & $313(74.2)$ \\
\hline Patients with no tender joints & 422 & $299(70.9)$ \\
\hline Patients with no restricted joints & 422 & $253(60)$ \\
\hline Patients with physician's VAS $=0$ & 422 & $239(56.6)$ \\
\hline Patients with ESR $<20 \mathrm{~mm} / \mathrm{h}$ & 398 & $321(80.7)$ \\
\hline Patients with normal CRP & 402 & $341(84.8)$ \\
\hline Patients with JADI Articular $=0$ & 419 & $324(77.3)$ \\
\hline Patients with JADI Extraarticular $=0$ & 419 & $364(86.9)$ \\
\hline
\end{tabular}

IQR interquartile range, JADAS Juvenile Arthritis Disease Activity Score, VAS visual analog scale, ESR erythrocyte sedimentation rate, CRP C-reactive protein, JADI Juvenile Arthritis Damage Index

${ }^{a}$ Data are number (percentage) unless otherwise indicated

${ }^{\mathrm{b}}$ Includes patients with inactive disease

the percentage of patients who were still receiving concomitant MTX therapy at cross-sectional visit was decreased from 73.7 to $47.4 \%$ and the percentage of patients who were taking systemic corticosteroids was diminished from 22 to $4.7 \%$. Only $2.6 \%$ of patients underwent intraarticular corticosteroid administration at cross-sectional visit. Of the 21 patients with systemic arthritis who were still receiving ETN, 15.8 and 9.5\% had ID by Wallace and JADAS criteria, respectively, and 23.8 and $28.6 \%$ had LDA by Magni-Manzoni and JADAS criteria, respectively.

Parent-reported outcomes obtained at cross-sectional visit through the completion of the JAMAR are summarized in Table 3. Around half of the patients had normal physical function and only $22.1 \%$ had impairment in HRQL, as demonstrated by a HRQL score 1SD above the mean of healthy children. HRQL impairment was more common in the physical than in the psychosocial domains (48.7\% vs. $19.7 \%) .46 \%$ of patients had a parent global assessment indicating good well-being and around half had no pain. The median (IQR) pain VAS score was 0 and, among patients who had a pain score $>0,68.8$, 23.6 and $7.6 \%$ had a score $\leq 1,1-5$ or $>5$, respectively. Three quarter of patients had no morning stiffness and around two third were judged by their parents as being in the state of remission. $82.7 \%$ of parents were satisfied with the current state of their child's illness. 
Table 3 Parent-reported outcomes at cross-sectional visit in patients still receiving ETN

\begin{tabular}{|c|c|c|}
\hline \multirow[b]{2}{*}{ Median (IQR) physical function score ${ }^{a}$} & \multicolumn{2}{|c|}{ No assessed } \\
\hline & 420 & $0(0 ; 3)$ \\
\hline No (\%) of patients with physical function score $=0$ & 420 & $220(52.4)$ \\
\hline Median (IQR) HRQL score ${ }^{\mathrm{b}}$ & 417 & $2(0 ; 5)$ \\
\hline No (\%) of patients with HRQL score > 1 DS above the mean of healthy children & 417 & $92(22.1)$ \\
\hline Median (IQR) HRQL PhH score ${ }^{c}$ & 417 & $1(0 ; 3)$ \\
\hline No (\%) of patients with HRQL PhH score > 1 DS above the mean of healthy children & 417 & $203(48.7)$ \\
\hline Median (IQR) HRQL PsH score ${ }^{c}$ & 417 & $1(0 ; 3)$ \\
\hline No (\%) of patients with HRQL PsH > 1 DS above the mean of healthy children & 417 & $82(19.7)$ \\
\hline Median (IQR) VAS well-being ${ }^{d}$ & 420 & $0.5(0 ; 3)$ \\
\hline No (\%) of patients with VAS well-being $=0$ & 420 & $193(46)$ \\
\hline Median (IQR) VAS pain ${ }^{d}$ & 420 & $0(0 ; 2,5)$ \\
\hline No (\%) of patients with VAS pain $=0$ & 420 & $215(51.2)$ \\
\hline No (\%) of patients with VAS pain $\leq 1$ & 420 & $289(68.8)$ \\
\hline No (\%) of patients with VAS pain from 1.5 to 5 & 420 & 99 (23.6) \\
\hline No (\%) of patients with VAS pain $>5$ & 420 & $32(7.6)$ \\
\hline Median (IQR) VAS disease activity ${ }^{d}$ & 420 & $0(0 ; 2)$ \\
\hline No (\%) of patients with VAS disease activity $=0$ & 420 & $224(53.3)$ \\
\hline No (\%) of patients with no morning stiffness & 420 & $319(76)$ \\
\hline \multicolumn{3}{|l|}{ Disease activity state } \\
\hline No (\%) of patients with remission & 421 & $286(67.9)$ \\
\hline No (\%) of patients with continued activity & 421 & $86(20.4)$ \\
\hline No (\%) of patients with disease flare & 421 & $49(11.6)$ \\
\hline No (\%) of parents satisfied with their child's illness outcome & 399 & $330(82.7)$ \\
\hline
\end{tabular}

IQR interquartile range, HRQL health-related quality of life, $\mathrm{PhH}$ Physical Health, $\mathrm{PsH}$ Psychosocial Health, VAS visual analog scale

${ }^{\text {a }}$ Score ranges from 0 (no disability) to 30 (maximum disability)

${ }^{\mathrm{b}} \mathrm{Score}$ ranges from 0 to 30 , higher scores indicate worse HRQL

'Score ranges $0-15$, higher scores indicate worse HRQL

${ }^{\mathrm{d}}$ All VAS range from 0 (best) to 10 (worst)

The achievement of disease remission was the leading cause of treatment discontinuation (52.4\% of patients), followed by treatment inefficacy (29\%), adverse events (21\%), and parent decision/lack of adherence (2.6\%). More than half of the patients lost to follow-up (55.8\%) were in clinical remission at the time of last visit. Of the 70 patients with systemic arthritis who were discontinued from ETN, 19 (27.1\%) and 45 (64.3\%) had treatment withdrawn for disease remission and inefficacy, respectively.

Among the 108 patients (10.4\%) who had not received MTX before ETN start, 35 were in the cross-sectional group and 73 were in the retrospective group. In the former group, the frequency of ID by Wallace and JADAS criteria was 25 and $28.6 \%$, respectively, and the frequency of LDA by Magni-Manzoni and JADAS criteria was 44.1 and $51.4 \%$, respectively. $71 \%$ per cent of the patients in the latter group had disease remission at treatment discontinuation or last follow-up visit.

One or more adverse events were reported for $27.8 \%$ of the patients. The most common were new onset or recurrence of uveitis (10.2\%), infections (6.6\%), injection site reactions $(4.4 \%)$, neuropsychiatric disorders (3.1\%), gastrointestinal disorders (2.4\%), hematological disorders (2.1\%), muco-cutaneous disorders $(1.9 \%)$, and pain at the injection site (1.8\%). Infectious complications were most frequently mild as in only 13 of 68 cases they led to treatment withdrawal. The side effects that led to treatment discontinuation in the 99 patients who were withdrawn from ETN for toxicity are presented in Table 4. Those that led to treatment discontinuation in more than $5 \%$ of patients were recurrence or new onset of uveitis, neuropsychiatric disorders, gastrointestinal disorders, infections, muco-cutaneous disorders, and hematologic disorders. Among these patients, the most common neuropsychiatric manifestations were behavioral disorders and headache, whereas urticaria and leukopenia were the most frequently reported mucocutaneous and hematologic disorders, respectively. Seven patients had injection site reaction or pain at injection site. Only 1 patient developed tuberculosis. 
Table 4 Adverse events that led to treatment discontinuation in 99 patients

\begin{tabular}{|c|c|}
\hline Adverse event & $\mathrm{N}$ \\
\hline Recurrent or new-onset uveitis & 38 \\
\hline Neuropsychiatric disorders & 21 \\
\hline Behavioral disorders & 7 \\
\hline Headache & 6 \\
\hline Mood disorders/difficulty concentrating & 4 \\
\hline Tics/unintentional movements & 2 \\
\hline Papilledema & 1 \\
\hline Hypoglossal nerve paralysis & 1 \\
\hline Gastrointestinal disorders & 15 \\
\hline Inflammatory bowel diseases & 10 \\
\hline Persistent hypertransaminasemia & 1 \\
\hline Abdominal pain & 1 \\
\hline Peritonitis anti-DNA positive & 1 \\
\hline Acute pancreatitis & 1 \\
\hline Nausea or vomiting & 1 \\
\hline Infection & 13 \\
\hline Recurrent herpes labialis & 2 \\
\hline Recurrent upper airway infections & 2 \\
\hline Fatal streptococcal sepsis & 1 \\
\hline Tuberculosis & 1 \\
\hline Varicella complicated by porpora fulminans and fasciitis & 1 \\
\hline Osteomyielitis & 1 \\
\hline Cellulitis & 1 \\
\hline Herpetic neuritis & 1 \\
\hline Herpes zoster & 1 \\
\hline CMV hepatitis & 1 \\
\hline Recurrent bronchitis & 1 \\
\hline Mucocutaneous disorders & 10 \\
\hline Urticaria & 4 \\
\hline Urticaria angioedema & 2 \\
\hline Cutaneous vasculitis & 2 \\
\hline Itch & 1 \\
\hline Anal Condylomatosis & 1 \\
\hline Haematological disorders & 5 \\
\hline Leukopenia & 3 \\
\hline Autoimmune thrombocytopenia & 1 \\
\hline Hypocomplementemia & 1 \\
\hline Injection site reactions & 4 \\
\hline Pain at injection site & 3 \\
\hline Malignancy & 2 \\
\hline Thyroid carcinoma & 1 \\
\hline Bladder carcinoma & 1 \\
\hline Others & 6 \\
\hline
\end{tabular}

Table 4 Adverse events that led to treatment discontinuation in 99 patients (Continued)

\begin{tabular}{ll}
\hline Persistent cervical lymphadenopathy & 2 \\
Autoimmune thyroiditis & 1 \\
Menometrorrhagia & 1 \\
Abortion & 1 \\
Renal lithiasis & 1 \\
\hline
\end{tabular}

The prevalence of uveitis was 52/314 (16.6\%) among patients who were started with ETN before December 2006 and 54/724 (7.5\%) among patients treated after January 2007. Furthermore, the 38 patients who had ETN discontinued due to the occurrence of uveitis had a median duration of ETN therapy longer than that of the entire patient cohort ( 3.4 and 2.5 years, respectively). Of the 10 patients who developed an inflammatory bowel disease, 4 had rheumatoid-factor negative polyarthritis, 2 oligoarthritis, 2 enthesitis-related arthritis, 1 rheumatoidfactor positive polyarthritis, and 1 psoriatic arthritis. Two patients had a malignancy while receiving ETN. The first was a 16-year old boy with persistent oligoarthritis who developed a thyroid carcinoma 1 year after the start of ETN. The disease duration at start of ETN was 3.7 years and the medication regimen before ETN initiation had included MTX and intra-articular corticosteroids. The second patient had a bladder carcinoma after 9.4 years of ETN administration. He was a boy with systemic arthritis who had a disease duration of 5.8 years at ETN start and had previously received MTX and systemic corticosteroids and intra-articular corticosteroids. The sole patient in the study cohort who died was a 5-year old child with systemic arthritis who was taking ETN and MTX and had a fulminant streptococcal sepsis, which developed as a complication of bilateral bronchopneumonia and pleurisy. He had previously received systemic corticosteroids and anakinra.

\section{Discussion}

Our study describes the experience with the use of ETN in Italian children with JIA. Because all but 3 centers that are part of the Italian Pediatric Rheumatology Study Group participated in the study and more than 95\% of patients included in the census underwent the crosssectional or retrospective assessments, the results of our survey are generalizable to all JIA patients who received ETN in Italy in the study period. To obtain a precise and reliable documentation of the disease status of children on ongoing ETN therapy, we applied a comprehensive set of physician-centered and parent-centered outcome measures. These data provide a benchmarking for future outcome comparisons with other cohorts of JIA patients under therapy with ETN or other biologic medications. 
We found that 41.8 to $48.6 \%$ of patients (depending of the definition used) who were still receiving ETN at the time of the census met the criteria for ID, $52.4 \%$ of patients who were discontinued from ETN had the medication stopped for clinical remission, and $55.8 \%$ of patients who were lost to follow-up were in clinical remission at last visit. Altogether, these findings concur with earlier reports that around half of JIA patients treated with ETN in standard clinical care are able to attain complete disease quiescence [12-14]. The strong disease-controlling potential of ETN was strengthened by the observation that in the cross-sectional sample 58.3 to $64 \%$ of patients had LDA and less than $15 \%$ had HDA. In addition, at crosssectional visit the proportion of patients who were taking concomitant MTX or systemic corticosteroid therapy was decreased from 73.7 to $47.4 \%$ and from 22 to $4.7 \%$, respectively, compared to treatment baseline. Only a small proportion of patients had evidence or articular or extraarticular damage.

These figures do not apply to the systemic arthritis subgroup, however, as there was a relatively greater proportion of patients with this JIA category among those who were discontinued or lost to follow-up than among those who were still taking ETN. A number of prior studies have shown that anti-TNF agents are less effective in the systemic subset of JIA [2, 3, 5, 12, 25-27]. This phenomenon has been attributed to interleukin (IL)-1 and IL-6 playing a greater pathogenetic role than TNF in systemic arthritis $[28,29]$. However, a sizeable proportion of our patients with systemic arthritis had ID or LDA at cross-sectional visit or had ETN discontinued for the achievement of disease remission. Some studies have suggested that TNF inhibitors may be effective in the later afebrile disease phase of systemic JIA, characterized by chronic arthritis $[4,30]$.

The results of parent-centered assessments at crosssectional visit corroborated the good disease status achieved by patients on continued ETN therapy, as $52.4 \%$ of them had normal physical function, a few had impairment in HRQL, $51.2 \%$ had no pain, $76 \%$ had no morning stiffness, and $67.9 \%$ were judged as having disease remission by the parent. In addition, $82.7 \%$ of parents were satisfied with their child's illness outcome. That around half of the patients had persistent pain is worrying, however, although only $7.6 \%$ had severe pain (i.e.,a pain level $>5$ on a $0-10$ VAS). A recent study found that a subgroup of JIA patients treated with TNF inhibitors had continuous pain despite good disease control [31]. These observations underscore the possible persistence of clinically significant pain in children treated with biologic agents, even when good disease control is achieved. Constant monitoring of pain and its determinants remains, therefore, a clinical priority in the current biologic era [32]. Notably, use of nonsteroidal anti-inflammatory drugs before ETN start was reported for only $58 \%$ of patients. This finding underscores the recent change in treatment practice related to these medications.

ETN was generally well tolerated, as clinically significant adverse events were reported for $27.8 \%$ of patients and the medication was discontinued for side effects in 99 patients (9.5\%). Overall, safety findings are consistent with those reported in other analyses of ETN in JIA $[2,4,26,33,34]$.

New-onset or recurrent uveitis was the adverse event most commonly recorded in the entire study sample and was most frequently responsible for treatment discontinuation. This finding is important as ocular inflammation has been postulated to be related to $\operatorname{ETN}[35,36]$. It should be taken into account, however, that our sample included a high proportion of children with earlyonset and ANA-positive disease, who have a high background risk of uveitis [37]. It has been hypothesized that ETN may not directly cause the development of uveitis, but the discontinuation of MTX upon successful arthritis control may pose the patient at risk [38]. Recent data have shown that MTX may prevent the onset of uveitis in children with JIA $[39,40]$. Nevertheless, many patients who had ETN discontinued for uveitis were likely switched to adalimumab and infliximab, which are nowadays recommended as second-line therapeutic option among patients with JIA and uveitis [41]. Notably, the frequency of uveitis was higher among patients treated in the earlier years of ETN use than in those who received ETN in the recent years $(16.6 \%$ versus $7.5 \%$ ), which suggests that the increased awareness of the possible relation of ETN with ocular inflammation might have led to decrease its prevalence. In addition, the 38 patients who had ETN discontinued because of the occurrence of uveitis had an average treatment duration longer than that of the entire patient cohort.

Previous studies have shown that the risk of infection is increased in children with JIA receiving ETN as compared to those treated with MTX [4, 26, 30, 42, 43]. Infection was the second most prevalent adverse event in our cohort, but only ranked fourth among the causes of treatment discontinuation for side effects. Indeed, infectious complications were most frequently mild as in only 13 of 68 cases they led to treatment withdrawal. It is noteworthy that only 1 patient had tuberculosis. One patient, who was taking ETN and MTX, died for a fulminant streptococcal sepsis. A similar case has been reported previously [44].

Neuropsychiatric symptoms were the second most frequent cause of treatment discontinuation for adverse events. A high frequency of psychiatric symptoms was reported in other studies of ETN in JIA [2, 4]. It is, however, difficult to dissect the responsibility of the 
medication itself from behavioral or mood disorders related to psychological disease burden or due to other medications, namely MTX [45]. Unfortunately, the design of our study did not allow us to establish whether they were related to ETN by investigating whether they had disappeared after its withdrawal.

Ten patients developed an inflammatory bowel disease (IBD). Although this phenomenon has been observed in other studies [46, 47], the relationship between ETN and gut inflammation is unclear. It has been speculated that an already established subclinical IBD may be activated by the use of TNF inhibitors [48]. Along this line, it is conceivable that children with enthesitis related arthritis are more susceptible to this phenomenon, as IBD are closely associated with spondyloarthropathies [49]. However, only 2 of the 10 patients who developed IBD in our cohort had this JIA category. The role of ETN in inducing IBD deserves further investigations.

In spite of earlier alarming data [50], there is currently no evidence of an increased incidence of malignancy among patients exposed to TNF inhibitors compared with MTX [36], and the increased risk of malignancy has been attributed to the autoimmune disease itself, rather than to treatment with TNF inhibitors [51-53]. Two malignancies were observed in our cohort, one thyroid carcinoma and one bladder carcinoma. This frequency is in the range of that observed in other studies of similar size $[38,54]$. These reports underscore the need to follow carefully JIA patients exposed to biologics and to continue long-term observation in adulthood. Further knowledge about risk profiles of these medications will be gained with for the accumulation of longterm data through national and international registries [55]. In 2011, the Pediatric Rheumatology INternational Trials Organisation (PRINTO) and the Pediatric Rheumatology European Society (PRES) created an international observational registry aimed to enroll children with JIA treated with methotrexate or biologic medications in any available formulations. This registry is named "Pharmacovigilance in JIA patients treated with biologic agents and/or MTX - Pharmachild" (European Union grant 260353) and is primarily aimed to evaluate the long-term safety of these therapeutic agents [56].

The findings of our study should be interpreted in the light of some potential caveats. Because our analysis was nonrandomized and observational, we cannot exclude that children still on treatment with ETN at the time of the study had a less severe disease than those discontinued from the medication or lost to follow-up. However, although the method used to assess the treatment outcome was different, the percentage of patients who were in clinical remission was similar between cross-sectional and retrospective populations. The disease status was only assessed at a single point of time, which precluded the possibility to establish whether clinical remission was sustained while receiving treatment or after its interruption, and how many patients were retreated. Adverse events were recorded through the retrospective review of patients' clinical charts. A retrospective analysis is subject to missing and possibly erroneous data. Thus, some side effects may be underreported. Notably, owing to the nonprospective nature of data collection, we could not calculate the crude rates of adverse events per 100 person-years of exposure. Furthermore, we acknowledge that attribution of adverse events was not done. The main strengths of our study are related to the size of the cohort, the methodology used for case ascertainment which minimized potential selection bias, and the detailed procedures used to assess patients on ongoing ETN therapy.

\section{Conclusions}

Our study shows that around half of children with nonsystemic JIA achieve complete disease quiescence under treatment with ETN. The medication was overall well tolerated, as only one quarter of patients experienced clinically significant adverse events and less than 10\% had treatment discontinued for toxicity.

\section{Acknowledgements}

The authors thank Drs. Maria Alessio (Napoli, Italy), Silvana Martino (Torino, Italy), and Angela Miniaci (Bologna, Italy) for including their patients in the census.

Permission for use of CHAQ and CHQ derived-material is granted through the scientific cooperation of the copyright holder ICORE of Woodside CA and HealthActCHQ Inc. of Boston, Massachusetts USA. All CHQ-related inquiries are directed to licensing@healthactchq.com.

\section{Funding}

The study was supported by an unconditioned research grant from Pfizer Inc.

Availability of data and materials

All data included in the manuscript are available upon request.

\section{Authors' contributions}

SV, SD, AC and AR interpreted and analyzed the patient data. FB made all statistical analyses. AR was a major contributor in writing the manuscript. All authors read and approved the final manuscript.

\section{Competing interests}

The authors declare that they have no competing interests.

\section{Consent for publication}

All authors have provided their consent for publication.

\section{Ethics approval and consent to participate}

Ethics approval was obtained at all participating centers and all parents or patients (as appropriate) have provided consent to participate in the study.

\section{Author details}

${ }^{1}$ Università degli Studi di Genova, Genova, Italy. ${ }^{2}$ Istituto Giannina Gaslini, Genova, Italy. ${ }^{3}$ Ospedale Pediatrico Bambino Gesù, Roma, Italy. ${ }^{4}$ Istituto Ortopedico Gaetano Pini, Milano, Italy. ${ }^{5}$ Ospedale Pediatrico Meyer, Firenze, Italy. ${ }^{6}$ Università degli Studi, Padova, Italy. ${ }^{7}$ IRCCS Burlo Garofolo, Trieste, Italy. ${ }^{8}$ Fondazione IRCCS Cà Granda - Ospedale Maggiore Policlinico, Milano, Italy. ${ }^{9}$ Azienda Ospedaliera Universitaria Policlinico G. Martino, Messina, Italy.

${ }^{10}$ Azienda Ospedaliero-Universitaria Policlinico Vittorio Emanuele, Catania, Italy. ${ }^{11}$ Università degli Studi di Brescia, Brescia, Italy. ${ }^{12}$ Ospedale di Orvieto, Orvieto, Italy. ${ }^{13}$ Università degli Studi di Chieti, Chieti, Italy. ${ }^{14}$ Seconda 
Università degli Studi di Napoli, Napoli, Italy. ${ }^{15}$ Azienda Ospedaliera Card. G. Panico, Tricase, Italy. ${ }^{16}$ Ospedale Regionale per le Microcitemie, Cagliari, Italy. ${ }^{17}$ Policlinico Universitario A. Gemelli, Roma, Italy. ${ }^{18}$ Ospedale A. Perrino, Brindisi, Italy. ${ }^{19}$ Università Politecnica delle Marche, Ancona, Italy. ${ }^{20}$ Ospedale Pediatrico G. Salesi, Ancona, Italy. ${ }^{21}$ Università degli Studi di Messina, Messina, Italy. ${ }^{22}$ Ospedale dei Bambini G. Di Cristina, Palermo, Italy. ${ }^{23}$ Università degli Studi di Pisa, Pisa, Italy. ${ }^{24}$ Arcispedale S.Maria Nuova, Reggio Emilia, Italy.

${ }^{25}$ IRCCS Fondazione Policlinico San Matteo, Pavia, Italy. ${ }^{26}$ Pediatria

II-Reumatologia, Istituto G. Gaslini, Largo G. Gaslini 5, 16147 Genova, Italy.

Received: 16 September 2016 Accepted: 24 November 2016 Published online: 20 December 2016

\section{References}

1. Lovell DJ, Giannini EH, Reiff A, Cawkwell GD, Silverman ED, Nocton JJ, et al. Etanercept in children with polyarticular juvenile rheumatoid arthritis. Pediatric Rheumatology Collaborative Study Group. N Engl J Med. 2000;342:763-9.

2. Quartier P, Taupin P, Bourdeaut F, Lemelle I, Pillet P, Bost M, et al. Efficacy of etanercept for the treatment of juvenile idiopathic arthritis according to the onset type. Arthritis Rheum. 2003:48:1093-101.

3. Horneff G, Schmeling H, Biedermann T, Foeldvari I, Ganser G, Girschick HJ, et al. The German etanercept registry for treatment of juvenile idiopathic arthritis. Ann Rheum Dis. 2004;63:1638-44.

4. Giannini EH, llowite NT, Lovell DJ, Wallace CA, Rabinovich CE, Reiff A, et al Long-term safety and effectiveness of etanercept in children with selected categories of juvenile idiopathic arthritis. Arthritis Rheum. 2009;60:2794-804.

5. Southwood TR, Foster HE, Davidson JE, Hyrich KL, Cotter CB, Wedderburn $L R$, et al. Duration of etanercept treatment and reasons for discontinuation in a cohort of juvenile idiopathic arthritis patients. Rheumatology (Oxford). 2011;50:189-95.

6. Halbig M, Horneff $\mathrm{G}$. Improvement of functional ability in children with juvenile idiopathic arthritis by treatment with etanercept. Rheumatol Int 2009:30:229-38.

7. Prince FH, Geerdink LM, Borsboom GJ, Twilt M, van Rossum MA, Hoppenreijs EP, et al. Major improvements in health-related quality of life during the use of etanercept in patients with previously refractory juvenile idiopathic arthritis. Ann Rheum Dis. 2010;69:138-42.

8. Klotsche J, Minden K, Thon A, Ganser G, Urban A, Horneff G. Improvement in health-related quality of life for children with juvenile idiopathic arthritis after start of treatment with etanercept. Arthritis Care Res (Hoboken). 2014;66:253-62.

9. Giannini EH, llowite NT, Lovell DJ, Wallace CA, Rabinovich CE, Reiff A, et al. Effects of long-term etanercept treatment on growth in children with selected categories of juvenile idiopathic arthritis. Arthritis Rheum. 2010;62:3259-64.

10. Billiau AD, Loop M, Le PQ, Berthet F, Philippet $P$, Kasran A, et al. Etanercept improves linear growth and bone mass acquisition in MTX-resistant polyarticular-course juvenile idiopathic arthritis. Rheumatology (Oxford). 2010:49:1550-8

11. Nielsen S, Ruperto N, Gerloni V, Simonini G, Cortis E, Lepore L, et al. Preliminary evidence that etanercept may reduce radiographic progression in juvenile idiopathic arthritis. Clin Exp Rheumatol. 2008;26:688-92.

12. Solari N, Palmisani E, Consolaro A, Pistorio A, Viola S, Buoncompagni A, et al. Factors associated with achievement of inactive disease in children with juvenile idiopathic arthritis treated with etanercept. J Rheumatol. 2013;40:192-200

13. Papsdorf V, Horneff G. Complete control of disease activity and remission induced by treatment with etanercept in juvenile idiopathic arthritis. Rheumatology (Oxford). 2011;50:214-21.

14. Otten MH, Prince FH, Armbrust W, ten Cate R, Hoppenreijs EP, Twilt M, et al. Factors associated with treatment response to etanercept in juvenile idiopathic arthritis. JAMA. 2011;306:2340-7.

15. Petty RE, Southwood TR, Manners P, Baum J, Glass DN, Goldenberg J, et al. International League of Associations for Rheumatology classification of juvenile idiopathic arthritis: second revision, Edmonton, 2001. J Rheumatol. 2004:31:390-2

16. Ravelli A, Viola S, Ruperto N, Corsi B, Ballardini G, Martini A. Correlation between conventional disease activity measures in juvenile chronic arthritis. Ann Rheum Dis. 1997;56:197-200.

17. Filocamo G, Davi S, Pistorio A, Bertamino M, Ruperto N, Lattanzi B, et al. Evaluation of 21-numbered circle and 10-centimeter horizontal line visual analog scales for physician and parent subjective ratings in juvenile idiopathic arthritis. J Rheumatol. 2010;37:1534-41.

18. Viola S, Felici E, Magni-Manzoni S, Pistorio A, Buoncompagni A, Ruperto N, et al. Development and validation of a clinical index for assessment of long-term damage in juvenile idiopathic arthritis. Arthritis Rheum. 2005;52:2092-102.

19. Filocamo G, Consolaro A, Schiappapietra B, Dalpra S, Lattanzi B, MagniManzoni S, et al. A new approach to clinical care of juvenile idiopathic arthritis: the Juvenile Arthritis Multidimensional Assessment Report. J Rheumatol. 2011;38:938-53.

20. Wallace CA, Giannini EH, Huang B, Itert L, Ruperto N. American College of Rheumatology provisional criteria for defining clinical inactive disease in select categories of juvenile idiopathic arthritis. Arthritis Care Res (Hoboken). 2011;63:929-36.

21. Magni-Manzoni S, Ruperto N, Pistorio A, Sala E, Solari N, Palmisani E, et al. Development and validation of a preliminary definition of minimal disease activity in patients with juvenile idiopathic arthritis. Arthritis Rheum. 2008:59:1120-7.

22. Consolaro A, Bracciolini G, Ruperto N, Pistorio A, Magni-Manzoni S, Malattia $C$, et al. Remission, minimal disease activity, and acceptable symptom state in juvenile idiopathic arthritis: defining criteria based on the juvenile arthritis disease activity score. Arthritis Rheum. 2012;64:2366-74.

23. Consolaro A, Ruperto N, Bracciolini G, Frisina A, Gallo MC, Pistorio A, et al. Defining criteria for high disease activity in juvenile idiopathic arthritis based on the juvenile arthritis disease activity score. Ann Rheum Dis. 2014;73:1380-3.

24. Consolaro A, Negro G, Gallo MC, Bracciolini G, Ferrari C, Schiappapietra B, et al. Defining criteria for disease activity states in non-systemic juvenile idiopathic arthritis based on a three-variable Juvenile Arthritis Disease Activity Score. Arthritis Care Res (Hoboken). 2014;66:1703-9.

25. Kimura Y, Pinho P, Walco G, Higgins G, Hummell D, Szer I, et al. Etanercept treatment in patients with refractory systemic onset juvenile rheumatoid arthritis. J Rheumatol. 2005;32:935-42.

26. Prince FH, Twilt M, ten Cate R, van Rossum MA, Armbrust W, Hoppenreijs $E P$, et al. Long-term follow-up on effectiveness and safety of etanercept in juvenile idiopathic arthritis: the Dutch national register. Ann Rheum Dis. 2009;68:635-41.

27. Tynjala $P$, Vahasalo $P$, Honkanen $V$, Lahdenne $P$. Drug survival of the first and second course of anti-tumour necrosis factor agents in juvenile idiopathic arthritis. Ann Rheum Dis. 2009;68:552-7.

28. Pascual V, Allantaz F, Arce E, Punaro M, Banchereau J. Role of interleukin-1 (IL-1) in the pathogenesis of systemic onset juvenile idiopathic arthritis and clinical response to IL-1 blockade. J Exp Med. 2005;201:1479-86.

29. De Benedetti F, Martini A. Is systemic juvenile rheumatoid arthritis an interleukin 6 mediated disease? J Rheumatol. 1998:25:203-7.

30. Lovell DJ, Reiff A, llowite NT, Wallace CA, Chon Y, Lin S-L, et al. Safety and efficacy of up to eight years of continuous etanercept therapy in patients with juvenile rheumatoid arthritis. Arthritis Rheum. 2008;58:1496-504.

31. Lomholt JJ, Thastum M, Herlin T. Pain experience in children with juvenile idiopathic arthritis treated with anti-TNF agents compared to non-biologic standard treatment. Pediatr Rheumatol Online J. 2013;11:21.

32. Consolaro A, Ravelli A. Paediatric rheumatology: Juvenile idiopathic arthritisare biologic agents effective for pain? Nat Rev Rheumatol. 2013;9:447-8.

33. Lovell DJ, Reiff A, Jones OY, Schneider R, Nocton J, Stein LD, et al. Longterm safety and efficacy of etanercept in children with polyarticular-course juvenile rheumatoid arthritis. Arthritis Rheum. 2006;54:1987-94.

34. Horneff G, De Bock F, Foeldvari I, Girschick HJ, Michels H, Moebius D, et al. Safety and efficacy of combination of etanercept and methotrexate compared to treatment with etanercept only in patients with juvenile idiopathic arthritis (JIA): preliminary data from the German JIA Registry. Ann Rheum Dis. 2009:68:519-25.

35. Wang F, Wang NS. Etanercept therapy-associated acute uveitis: a case report and literature review. Clin Exp Rheumatol. 2009;27:838-9.

36. Kakkassery $\mathrm{V}$, Mergler S, Pleyer U. Anti-TNF-alpha treatment: a possible promoter in endogenous uveitis? observational report on six patients: occurrence of uveitis following etanercept treatment. Curr Eye Res. 2010;35:751-6.

37. Calandra S, Gallo MC, Consolaro A, Pistorio A, Lattanzi B, Bovis F, et al. Female sex and oligoarthritis category are not risk factors for uveitis in italian children with juvenile idiopathic arthritis. J Rheumatol. 2014:41:1416-25.

38. Klotsche J, Niewerth M, Haas JP, Huppertz HI, Zink A, Horneff G, et al. Long term safety of etanercept and adalimumab compared to methotrexate in patients with juvenile idiopathic arthritis (JIA). Ann Rheum Dis. 2016;75:855-61. 
39. Papadopoulou C, Kostik M, Bohm M, Nieto-Gonzalez JC, GonzalezFernandez Ml, Pistorio A, et al. Methotrexate therapy may prevent the onset of uveitis in juvenile idiopathic arthritis. J Pediatr. 2013;163:879-84.

40. Kostik MM, Gaidar EV, Hynnes AY, Dubko MF, Masalova W, Snegireva LS, et al. Methotrexate treatment may prevent uveitis onset in patients with juvenile idiopathic arthritis: experiences and subgroup analysis in a cohort with frequent methotrexate use. Clin Exp Rheumatol. 2016;34:714-8.

41. Levy-Clarke G, Jabs DA, Read RW, Rosenbaum JT, Vitale A, van Gelder RN. Expert panel recommendations for the use of anti-tumor necrosis factor biologic agents in patients with ocular inflammatory disorders. Ophthalmol. 2014;121:785-96.

42. Davies R, Southwood TR, Kearsley-Fleet L, Lunt M, Hyrich KL. Medically significant infections are increased in patients with juvenile idiopathic arthritis treated with etanercept: results from the British Society for Paediatric and Adolescent Rheumatology Etanercept Cohort Study. Arthritis Rheumatol. 2015;67:2487-94.

43. Beukelman T, Xie F, Baddley JW, Chen L, Delzell E, Grijalva CG, et al. Brief report: incidence of selected opportunistic infections among children with juvenile idiopathic arthritis. Arthritis Rheum. 2013;65:1384-9.

44. Renaud C, Ovetchkine P, Bortolozzi P, Saint-Cyr C, Tapiero B. Fatal group A Streptococcus purpura fulminans in a child receiving TNF-alpha blocker. Eur J Pediatr. 2011:170:657-60.

45. van Dijkhuizen EH, Pouw JN, Scheuern A, Hugle B, Hardt S, Ganser G, et al. Methotrexate intolerance in oral and subcutaneous administration in patients with juvenile idiopathic arthritis: a cross-sectional, observational study. Clin Exp Rheumatol. 2016;34:148-54.

46. Wiegering V, Morbach H, Dick A, Girschick HJ. Crohn's disease during etanercept therapy in juvenile idiopathic arthritis: a case report and review of the literature. Rheumatol Int. 2010;30:801-4.

47. van Dijken TD, Vastert SJ, Gerloni VM, Pontikaki I, Linnemann K, Girschick H, et al. Development of inflammatory bowel disease in patients with juvenile idiopathic arthritis treated with etanercept. J Rheumatol. 2011;38:1441-6.

48. Dallocchio A, Canioni D, Ruemmele F, Duquesne A, Scoazec JY, Bouvier R, et al. Occurrence of inflammatory bowel disease during treatment of juvenile idiopathic arthritis with etanercept: a French retrospective study. Rheumatology (Oxford). 2010;49:1694-8.

49. Tse SML, Petty RE. Enthesitis related arthritis. In: Petty RE, Laxer RM, Lindsley $\mathrm{CB}$, Wedderburn LR, editors. Textbook of pediatric rheumatology. 7th ed. Philadelphia: Elsevier; 2016. p. 238-55.

50. Diak P, Siegel J, La GL, Choi L, Lemery S, McMahon A. Tumor necrosis factor alpha blockers and malignancy in children: forty-eight cases reported to the Food and Drug Administration. Arthritis Rheum. 2010;62:2517-24.

51. Ruperto N, Martini A. Juvenile idiopathic arthritis and malignancy. Rheumatology (Oxford). 2014;53:968-74.

52. Beukelman T, Haynes K, Curtis JR, Xie F, Chen L, Bemrich-Stolz CJ, et al. Rates of malignancy associated with juvenile idiopathic arthritis and its treatment. Arthritis Rheum. 2012;64:1263-71.

53. Simard J, Neovius M, Hagelberg S, Askling J. Juvenile idiopathic arthritis and risk of cancer: a nationwide cohort study. Arthritis Rheum. 2010;62:3776-82.

54. Horneff G, Foeldvari I, Minden K, Moebius D, Hospach T. Report on malignancies in the German juvenile idiopathic arthritis registry. Rheumatology (Oxford). 2011;50:230-6.

55. Ruperto N, Martini A. International research networks in pediatric rheumatology: the PRINTO perspective. Curr Opin Rheumatol. 2004;16(5):566-70.

56. Consolaro A, Morgan EM, Giancane G, Rosina S, Lanni S, Ravelli A. Information technology in pediatric rheumatology. Clin Exp Rheumatol. 2016:34 Suppl 101:S11-6.

\section{Submit your next manuscript to BioMed Central and we will help you at every step:}

- We accept pre-submission inquiries

- Our selector tool helps you to find the most relevant journal

- We provide round the clock customer support

- Convenient online submission

- Thorough peer review

- Inclusion in PubMed and all major indexing services

- Maximum visibility for your research

Submit your manuscript at www.biomedcentral.com/submit

) Biomed Central 